\title{
Optimizing output regulation for a class of underactuated LPV systems
}

\author{
Dario Corona $^{(1)}$, Andrea Cristofaro ${ }^{(1), *}$ and Damiano Rotondo ${ }^{(2)}$
}

\begin{abstract}
In this paper the problem of optimizing the output regulation of an underactuated LPV system is considered. When the system is underactuated, only a subset of the outputs can be arbitrarily controlled, and the remaining ones are constrained. Having identified a special class of LPV systems admitting steady-states, the problem of finding the input that minimizes a cost function of the overall output tracking error has been investigated. Moreover, it has been shown how such solution is related to the inputs associated to the singularly optimal regulation of each output.
\end{abstract}

\section{INTRODUCTION}

Redundancy is a key feature in a control system, as it provides additional degrees of freedom in the usage of available devices, e.g. actuators, effectors and sensors [17]. In [31], input redundant linear systems (described as a matrix quadruple $\{A, B, C, D\}$ ) are classified as strongly redundant if the columns of matrix $\left[\begin{array}{ll}B^{\prime} & D^{\prime}\end{array}\right]^{\prime}$ are not all linearly independent (which is equivalent to say that at least one of the inputs can always be exactly replaced by a suitable combination of the remaining inputs), and weakly redundant otherwise. There are several ways to take advantage of strong redundancy: among others, well established setups are optimization of system performances [30] [11], compensation for saturations and other input limitations [3] [15], and fault tolerance [4] [8]. Conversely, in the context of weak redundancy, the great majority of results aims at using the additional degrees of freedom in the selection of the control law to optimize the actuator states while keeping the same output performances [13], [28], [7], [12], [20], [32]. The results reported in this paper fit instead the dual problem of output redundancy or dual redundancy, that is the opposite setup where a redundant number of outputs is present. In this regard, we have also two different notions of dual redundancy. A plant is referred to as strongly output redundant when the output matrix $C$ is not full rank, this corresponding to a system equipped with a surplus of sensors. We will instead refer to a plant as weakly output redundant when the number of outputs is larger than the number of inputs, namely $m=\operatorname{rank}(B)<\operatorname{rank}(C)=p$.

Strong dual redundancy can be exploited to enhance antiwindup schemes for output saturated closed loops [27] by enlarging stability regions and reducing the compensation

(1) School of Science and Technology (Mathematics Division), University of Camerino, Camerino (MC), Italy. \{dario.corona, andrea.cristofaro\}eunicam.it

(2) Centre for Autonomous Marine Operations and Systems (AMOS), Department of Engineering Cybernetics, Norwegian University of Science and Technology, Trondheim, Norway. damiano.rotondo@yahoo. it

* Corresponding author.

$\diamond$ Authors are listed in alphabetical order.

- This work has been supported by the Research Council of Norway through the Centres of Excellence funding scheme (ref. 223254 - AMOS). D. Rotondo is also supported by the ERCIM Alain Bensoussan Fellowship programme. load; moreover, strong redundancy is an useful tool for accommodating sensor faults, and therefore it is a setup that can be successfully adopted to improve safety and reliability of systems for many applications such as dynamic positioning of ships [24]. Another possible way to take advantage of a strongly redundant output setting is to consider the problem of optimizing the output injected signal for an observer [9]. Weak dual redundancy is a typical characteristic of under-actuated systems. Classical examples of underactuated mechanical systems can found in the framework of underwater robotics, such as ROVs and AUVs [10] [19]. It is well known that, in the presence of a under-actuated system, the overall control action is in general insufficient to shape, simultaneously and independently, the response of all the outputs. On the other hand giving priority to one output might severely penalize the performance of the others, and the magnitude of such penalization depends on many different factors, such as given output references, functional controllability space structure, and system dimension. For single input linear plants with constant references, a systematic method to optimize the overall output tracking error has been proposed in [5] and then extended in [6] for the case of periodic and quasiperiodic references. A simple framework is weighting uniformly the error of each output, i.e. seeking for the control input the minimizes the norm of the tracking error. However, other solutions are possible, corresponding to different priorities in the outputs; this can be naturally addressed by introducing more general cost functions.

The aim of this paper is to apply the same ideas to the more challenging context of linear parameter varying (LPV) systems. LPV systems are a formalism which is established as a standard for gain-scheduling analysis and controller synthesis [29]. Many nonlinear systems can be converted into a quasi-LPV form by embedding the nonlinearities within appropriate varying parameters [18] (quasi refers to the dependence of the varying parameters on endogenous signals, i.e. the state and/or the input [22]), such that their control can be performed using an extension of linear techniques [1]. The LPV framework has been applied successfully in many applications, e.g. [25], [26], and has been validated by several experiments and high-fidelity simulations [16].

The outline of the paper is as follows. The formal problem statement is given in Section II, while in Section III a class of LPV systems admitting steady-states is analyzed. Input design for the optimal output regulation of such class of LPV systems is proposed in Section IV . Section V presents some remarks on the existence of neutral reference spaces, i.e. sets of references with the same optimal input. The superposition of the optimized input with an error feedback controller is investigated in Section VI. Finally, in Section VII, numerical simulations illustrate the application of the proposed results. 


\section{Statement of the Problem}

Let $\Sigma(\theta)=(A(\theta), b(\theta), C(\theta))$ be a (tall) LPV system with $A(\theta) \in \mathbb{R}^{n \times n}, b(\theta) \in \mathbb{R}^{n \times 1}$ and $C(\theta) \in \mathbb{R}^{p \times n}, n \geq p>1$

$$
\begin{aligned}
& \dot{x}(t)=A(\theta(t)) x(t)+b(\theta(t)) u(t) \\
& y(t)=C(\theta(t)) x(t)
\end{aligned}
$$

The vector $\theta \in \mathbb{R}^{z}, z \geq 1$, represents varying parameters, and can take values in a closed and bounded set $\Theta \subset \mathbb{R}^{z}$.

The vector $y(t)=\left[y_{1}(t) y_{2}(t) \ldots y_{p}(t)\right]^{T} \in \mathcal{C}\left(\mathbb{R}, \mathbb{R}^{p}\right)$ is the output of the system under the input $u(t)$, which it is assumed to belong to the set of piecewise continuous and bounded functions $\mathcal{U}$.

It is assumed that the origin is an exponentially stable equilibrium state for arbitrary time variations of the varying parameter $\theta(t)$ when $u(t)=0$, i.e. there exists a Lyapunov function of the form

$$
V(x(t), \theta(t))=x(t)^{T} P(x(t), \theta(t)) x(t)
$$

with the property that $P(x(t), \theta(t))^{T}=P(x(t), \theta(t))=$ $P(\varsigma x(t), \theta(t))>0, \forall \theta \in \Theta, \forall x \in \mathbb{R}^{n}, \forall \varsigma>0$ [21]. Notice that the Lyapunov condition $\dot{V}(x(t), \theta(t))<0$ for (2) reads:

$$
A(\theta)^{T} P(x, \theta)+P(x, \theta) A(\theta)+\dot{P}(x, \theta)<0
$$

Let $r(t)=\left[r_{1}, \ldots, r_{p}\right]^{T} \in \mathbb{R}^{p}$ be a constant reference vector and let denote with $e(t)=y(t)-r$ the tracking error vector. Suppose to have as objective the regulation of the outputs in order to reach the constant set points $r$. This can be formulated formally as the following control problem.

Output Tracking Problem: Find an input $u \in \mathcal{U}$ that guarantees the fulfillment of the following asymptotic condition

$$
\lim _{t \rightarrow \infty} e(t)=0 \in \mathbb{R}^{p}
$$

Since the plant $\Sigma(\theta)$ in (1) is under-actuated, the existence of a control $u(t)$ that leads $e(t) \rightarrow 0$ is not granted and can not be expected in general, even for frozen values of $\theta$, i.e. LTI (Linear Time Invariant) systems. For this reason, we are interested in investigating the following relaxed problem.

Relaxed Output Tracking Problem: Let $\|\cdot\|: \mathbb{R}^{p} \rightarrow$ $\mathbb{R}$ be a norm in the output space $\mathbb{R}^{p}$. Given an underactuated single-input LPV system as in (1), find $u \in \mathcal{U}$ that minimizes the steady-state tracking error, i.e. that minimizes the following functional

$$
J(u, r)=\lim _{t \rightarrow \infty}\|e(t)\|
$$

For LPV systems, the fulfilment of a constant steady output

$$
\lim _{t \rightarrow \infty} y(t)=y_{\infty} \neq 0
$$

is not granted in general. Our purpose is to characterize a special subset of LPV systems that admits a reachable space of asymptotically stable outputs. Given this conditions on the LPV system, we will search the solution of the relaxed output tracking problem in the space of solutions that will lead to constant stable outputs.

\section{Steady State Characterization}

\section{A. Non-unique solution of the relaxed tracking problem}

The asymptotic nature of the functional and the stability of the system lead to a non-unique minimum of (4). Indeed, the following proposition holds.

Proposition 1: Define the following subset of $\mathcal{U}$

$$
\mathcal{U}_{0}=\left\{u \in \mathcal{U} \text { s.t. } \lim _{t \rightarrow+\infty} u(t)=0\right\}
$$

Given $u_{1}, u_{2} \in \mathcal{U}$, let $y^{1}(t), y^{2}(t)$ be the corresponding outputs for the system (1). If $u_{1}(t)-u_{2}(t) \in \mathcal{U}_{0}$, then $y^{1}(t) \rightarrow y^{2}(t)$.

Proof: See Property 4 in [23].

As a consequence the hypothetical optimal solution $u^{*} \in$ $\mathcal{U}$ is not unique. Indeed, suppose that $\exists u^{*} \in \mathcal{U}$ such that $J\left(u^{*}\right)$ is the minimum reachable value. Then, for every $\bar{u}=$ $u^{*}+u^{\prime}$, with $u^{\prime} \in \mathcal{U}_{0}, u^{\prime} \neq 0, J(\bar{u})=J\left(u^{*}\right)$ despite $\bar{u} \neq u^{*}$. To overcome this issue, let us define the equivalence relation

$$
u_{1} \sim u_{2} \Longleftrightarrow u_{1}-u_{2} \in \mathcal{U}_{0}
$$

its relative quotient set

$$
\tilde{\mathcal{U}}=\mathcal{U} / \sim
$$

and let us denote the distinct elements of $\tilde{\mathcal{U}}$ with $\tilde{u}$. Due to Proposition 1, every two inputs $u_{1}, u_{2} \in \mathcal{U}$ with $u_{1} \sim$ $u_{2}$ drive asymptotically the system to the same trajectory. Accordingly, if for a given $u \in \mathcal{U}$ the value of (4) is welldefined, then it is so $\forall v \in \mathcal{U}$ with $v \sim u$. In this regard, as this paper concerns the minimization of the asymptotic tracking error (4), one can restricted the focus on the functional $J(\tilde{u}, r)$ evaluated on the class of equivalent inputs $\tilde{u} \in \tilde{\mathcal{U}}$. Moreover, $\tilde{0}$ will denote the null element of $\tilde{\mathcal{U}}$.

\section{B. Existence of Equilibrium States}

For a general LPV system as in (1), the value of (4) is not well-defined for every $\tilde{u} \neq \tilde{0}$. In the following, a special class of LPV systems that admit a non-zero control $\tilde{u} \in \tilde{\mathcal{U}}$ with a well-defined finite value of $J(\tilde{u}, r)$ will be characterized.

Definition 1: $\tilde{\mathcal{U}}_{\infty}^{y} \subset \tilde{\mathcal{U}}$ is the set of inputs that lead to a stable steady output $y_{\infty}$

$$
\tilde{\mathcal{U}}_{\infty}^{y}=\left\{\tilde{u} \in \tilde{\mathcal{U}}: \exists \lim _{t \rightarrow \infty} y(t)=y_{\infty} \in \mathbb{R}^{p}\right\} \subset \tilde{\mathcal{U}}
$$

By definition, if $\tilde{u} \in \tilde{\mathcal{U}}_{\infty}^{y}$, a well defined value of (4) is granted for every reference vector $r \in \mathbb{R}^{p}$ and for every norm function $\|\cdot\|: \mathbb{R}^{p} \rightarrow \mathbb{R}$, such that an optimal input (in the sense of minimizing (4)) can be searched in $\tilde{\mathcal{U}}_{\infty}^{y}$. Even though $\tilde{\mathcal{U}}_{\infty}^{y}=\tilde{\mathcal{U}}_{0}$ for most LPV systems, some sufficient conditions for an LPV system as in (1) to have a non-trivial set $\tilde{\mathcal{U}}_{\infty}^{y} \neq \tilde{\mathcal{U}}_{0}$ can be found.

If the LPV system in (1) has a constant output matrix $C(\theta)=C$, then the existence of an equilibrium state $x_{\infty}$ will guarantee the existence of an equilibrium output $y_{\infty}=C x_{\infty}$. Hence, sufficient conditions for the existence of equilibrium states $x_{\infty}$ different from the origin can be found.

Definition 2: An under-actuated LPV system is Steady Stable if

$$
\tilde{\mathcal{U}}_{\infty}^{x}=\left\{\tilde{u} \in \tilde{\mathcal{U}}: \exists \lim _{t \rightarrow \infty} x(t)=x_{\infty} \in \mathbb{R}^{n}\right\} \neq \tilde{\mathcal{U}}_{0}
$$


In other words, an LPV system is Steady Stable if there exists an input function $\tilde{u} \neq \tilde{0}$ that leads to a stable steady state. A stable steady state has to be an equilibrium state under the input $\tilde{u}$, so the next theorem provides sufficient conditions for the existence of equilibrium states different from the origin.

Theorem 1: Let $\Sigma(\theta)=(A(\theta), b(\theta), C(\theta))$ be an exponentially stable ${ }^{1}$ single-input under-actuated LPV system. If $\exists k \in \mathbb{R}^{n}, k \neq 0$ and a continuous function $\phi: \Theta \rightarrow \mathbb{R}$ such that

$$
-A(\theta)^{-1} b(\theta)=k \phi(\theta) \quad \forall \theta \in \Theta
$$

then there exist functions $u(\theta)$ such that $x_{\infty}=\alpha k, \alpha \in \mathbb{R}$, are equilibrium states, i.e.

$$
A(\theta) x_{\infty}+b(\theta) u(\theta)=0 \quad \forall \theta \in \Theta
$$

Proof: By choosing

$$
x_{\infty}=\alpha k=-A(\theta)^{-1} b(\theta) \frac{\alpha}{\phi(\theta)}
$$

and $u(\theta)=\frac{\alpha}{\phi(\theta)}$, the following is obtained

$$
\begin{aligned}
& A(\theta) x_{\infty}+b(\theta) u(\theta)= \\
& =-A(\theta) A(\theta)^{-1} b(\theta) \frac{\alpha}{\phi(\theta)}+b(\theta) \frac{\alpha}{\phi(\theta)}=0
\end{aligned}
$$

Notice that all the possible equilibrium states lie on a line in $\mathbb{R}^{n}$. This fact provides a geometric interpretation of the vector $k$, which is the direction of this line.

Remark 1: In Theorem 1, the function $\phi(\theta)$ can be chosen such that $\phi(\theta)>0 \forall \theta \in \Theta$. Indeed, if $\exists \bar{\theta} \in \Theta$ such that $\phi(\bar{\theta})=0$, then $x_{\infty}=k \phi(\bar{\theta})=0$ and $k$ can be chosen as $k=0 \in \mathbb{R}^{p}$, therefore obtaining $\phi(\theta) \equiv 1$. On the other hand, if $\exists \bar{\theta} \in \Theta$ such that $\phi(\theta)<0$, then $x_{\infty}=k \phi(\bar{\theta})=$ $-k(-\phi(\bar{\theta}))$. Choosing $\bar{k}=-k \in \mathbb{R}^{p}$ and $\bar{\phi}(\theta)=-\phi(\theta)$, a new pair $(\bar{k}, \bar{\phi})$ is obtained such that (10) holds and $\bar{\phi}(\theta)>$ $0 \forall \theta \in \Theta$.

\section{Reachability and Stability of the Equilibrium States}

Theorem 1 provides sufficient conditions for the existence of equilibrium states $x_{\infty}$. Hereafter, the exponential stability of these states is derived from the exponential stability of the origin for $u(t)=0$.

Theorem 2: Suppose that $\exists k \in \mathbb{R}^{n}$ and a real function $\phi$ : $\Theta \rightarrow \mathbb{R}$ such that (10) holds. If the origin is an exponentially stable equilibrium state for the LPV system (1) with $u(t)=$ 0 , then all the equilibrium states $x_{\infty}=\alpha k$ are exponentially stable with the inputs $u(\theta)=\alpha \phi(\theta)^{-} 1$.

Proof: Consider the Lyapunov function

$$
\Psi(x, \theta)=(x-\alpha k)^{T} P(x, \theta)(x-\alpha k)
$$

for which the derivative is given by

$$
\begin{aligned}
\dot{\Psi}(x, u, \theta) & =2(x-\alpha k)^{T} P(x, \theta)(A(\theta) x+b(\theta) u) \\
& +(x-\alpha k)^{T} \dot{P}(x, \theta)(x-\alpha k)
\end{aligned}
$$

From the hypothesis, it follows that

$$
u=\frac{\alpha}{\phi(\theta)} \Longrightarrow \alpha k=-A(\theta)^{-1} b(\theta) u(\theta)
$$

${ }^{1}$ The stability of $\Sigma(\theta)$ assures the invertibility of the state matrix $A(\theta)$. which means that (13) can be rewritten as

$$
\begin{gathered}
\dot{\Psi}(x, u, \theta)=2(x-\alpha k)^{T} P(x, \theta) A(\theta)\left(x+A(\theta)^{-1} b(\theta) u(\theta)\right) \\
+(x-\alpha k)^{T} \dot{P}(x, \theta)(x-\alpha k) \\
=2(x-\alpha k)^{T} P(x, \theta) A(\theta)(x-\alpha k) \\
+(x-\alpha k)^{T} \dot{P}(x, \theta)(x-\alpha k) \\
=(x-\alpha k)^{T}\left(A(\theta)^{T} P(x, \theta)+P(x, \theta) A(\theta) \cdots\right. \\
\cdots+\dot{P}(x, \theta))(x-\alpha k)
\end{gathered}
$$

Hence, from (3), it follows that $\dot{\Psi}(x, u, \theta)<0 \forall x \neq \alpha k$, which implies that the states $x_{\infty}=\alpha k$ are exponentially stable for the inputs $u=\alpha \phi(\theta)^{-1}$.

As consequence, using Theorem 1 and Theorem 2, if (3) and (10) hold, the LPV system $\Sigma(\theta)=(A(\theta), b(\theta), C(\theta))$ is Steady Stable. Moreover, let us consider an LPV system $\Sigma(\theta)=(A(\theta), b(\theta), C)$ where the output matrix does not depend on the varying parameter $\theta$. In this case a steady state $x_{\infty}$ leads to a steady output $y_{\infty}$, hence $\tilde{\mathcal{U}}_{\infty}^{x} \subset \tilde{\mathcal{U}}_{\infty}^{y}$, so $\tilde{\mathcal{U}}_{\infty}^{x} \neq \tilde{\mathcal{U}}_{0}$ implies $\tilde{\mathcal{U}}_{\infty}^{y} \neq \tilde{\mathcal{U}}_{0}$. The next corollary summarizes all the previous results.

Corollary 1: Let $\Sigma(\theta)=(A(\theta), b(\theta), C)$ be an underactuated single input LPV system.

If

- $\exists k \in \mathbb{R}^{n}, k \neq 0$ and a continuous function $\phi: \Theta \rightarrow \mathbb{R}$ such that (10) holds

- $\exists P(x, \theta)=P(x, \theta)^{T} \in \mathbb{R}^{n \times n}$ such that $\forall \theta \in \Theta, \forall x \in$ $\mathbb{R}^{n}, \forall \varsigma>0 P(x, \theta)=P(\varsigma x, \theta)$ and (3) holds then

$$
\tilde{\mathcal{U}}_{\infty}^{y} \neq \tilde{\mathcal{U}}_{0}
$$

\section{Optimal InPUT Synthesis}

In this section, the input $\tilde{u} \in \tilde{\mathcal{U}}$ that minimizes (4) is found for different norm functions $\|\cdot\|: \mathbb{R}^{p} \rightarrow \mathbb{R}$. The two hypothesis of Corollary 1 are assumed to be satisfied, such that the optimal input can be found in $\tilde{\mathcal{U}}_{\infty}^{y} \neq \tilde{\mathcal{U}}_{0}$. Let us define

$$
w=C k \in \mathbb{R}^{p},
$$

which is the direction of all the possible equilibrium outputs.

\section{A. Euclidean Distance}

Theorem 3: Let $\Sigma(\theta)=(A(\theta), b(\theta), C)$ be an underactuated LPV system such that (3) and (10) hold, and let us consider the norm function

$$
J(u)=\lim _{t \rightarrow \infty} \frac{1}{2} e(t)^{T} e(t)
$$

If $w=C k \neq 0 \in \mathbb{R}^{p}$, then the input

$$
u^{*}(\theta)=\frac{1}{\phi(\theta)} \frac{w^{T}}{w^{T} w} r
$$

is a minimum for (17).

Proof: A consequence of Theorem 1 is that the reachable stable outputs of the system are given by

$$
y_{\infty}=C \alpha k=\alpha w
$$

The functional in (17) can be written as a function of $\alpha$

$$
J(u)=J(\alpha)=\frac{1}{2}(\alpha w-r)^{T}(\alpha w-r)
$$


Deriving with respect to $\alpha$ and setting the derivative equal to zero, the following is obtained

$$
\alpha=\frac{w^{T}}{w^{T} w} r
$$

So the optimum input is given by (18).

Remark 2: If $\alpha=w_{i}^{-1} r_{i}$, then

$$
u^{i}=\frac{w_{i}^{-1} r_{i}}{\phi(\theta)}
$$

and, consequently, $y_{i}(t) \rightarrow \alpha w_{i}=r_{i}$. Hence, the different $u^{i}, i=1, \ldots, p$, are the inputs that ensure, respectively, $y_{i}(t) \rightarrow r_{i}$. Let

$$
\lambda_{i}=\frac{w_{i}^{2}}{w^{T} w} \in[0,1]
$$

then

$$
u^{*}(\theta)=\frac{1}{\phi(\theta)} \frac{w^{T} r}{w^{T} w}=\sum_{i=1}^{p} \frac{1}{\phi(\theta)} \frac{w_{i}^{2}}{w^{T} w} w_{i}^{-1} r_{i}=\sum_{i=1}^{p} \lambda_{i} u^{i}
$$

Moreover, it is straightforward to verify that $\sum_{i=1}^{p} \lambda_{i}=1$., which means that (18) can be seen as a convex combination of the different inputs $u^{i}$.

\section{B. Weighted Euclidean Distance}

A simple norm that allows to assign different priorities is a weighted Euclidean squared norm. Let

$$
\Gamma=\operatorname{diag}\left(\gamma_{1}, \ldots, \gamma_{p}\right) \quad \gamma_{i} \geq 0 \quad \forall i=1, \ldots, p
$$

be the weighting matrix. In this case, the error function to minimize is the quadratic expression

$$
J(u)=\lim _{t \rightarrow \infty} e(t)^{T} \Gamma e(t)
$$

The optimal solution $u^{*}$ can be obtained with the same procedure as for the standard Euclidean squared norm. The solution $u^{*}$ is

$$
u^{*}=\frac{1}{\phi(\theta)} \frac{w^{T} \Gamma r}{w^{T} \Gamma w} .
$$

and it can be viewed as a convex combination of the different $u^{i}$ given by (20), with coefficients

$$
\lambda_{i}=\frac{w_{i}^{2} \gamma_{i}}{w^{T} \Gamma w}
$$

\section{Geometric Interpretation}

For a Steady Stable LPV system with $C(\theta)=C$, a useful geometric interpretation of the solution can be achieved.

In this case, the reachable steady states can be written as $x_{\infty}=\alpha k$. As consequence, the reachable steady outputs are

$$
y_{\infty}=\alpha C k=\alpha w
$$

which is a line in $\mathbb{R}^{p}$ (see Fig. 1).

Moreover, in the case of squared euclidean distance norm (17), the optimal input (18) leads to the optimal output

$$
y_{\infty}^{*}=\frac{w^{T} r}{w^{T} w} w
$$

that is the projection of the reference vector $r$ on the line $y_{\infty}=\alpha w$, as shown in Fig. 1 .

A similar interpretation can be given in the case of weighted euclidean distance (24).

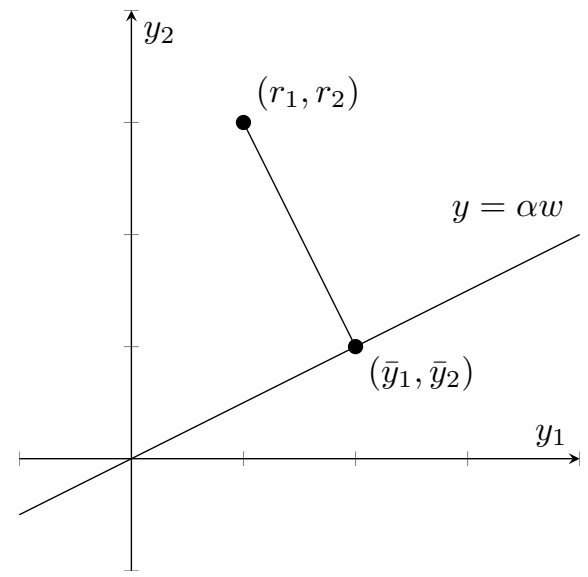

Fig. 1. A geometric representation of the solution for $p=2$

\section{Neutral Reference Spaces}

The geometric interpretation of the solution $y_{\infty}^{*}$ suggest the existence of sets of reference vectors characterized by the same optimum output. Indeed, we can expect that all the reference vectors with the same projection on the line $y=\alpha w$ share the same optimal input.

More rigorously, let $\Sigma(\theta)=(A(\theta), b(\theta), C)$ be a Steady Stable LPV system and $J(u)$ as in (4) a given cost function. Let us denote with $\mathcal{S}_{J}: \mathbb{R}^{p} \rightarrow \tilde{\mathcal{U}}$ the mapping that associate to a given reference vector the corresponding optimal input for the cost function $J(\tilde{u}, r)$. In the case of squared euclidean distance and weighted euclidean distance, $\mathcal{S}_{J}(r)$ is given by (18) and (25), respectively. Let us define the neutral space of a given $\bar{r}$ as

$$
\mathcal{N}_{J}(\bar{r})=\left\{r \in \mathbb{R}^{p}: \mathcal{S}_{J}(r)=\mathcal{S}_{J}(\bar{r})\right\}
$$

In the case of weighted euclidean norm function, a characterization of $\mathcal{N}_{J}(\bar{r})$ can be given as follows.

Proposition 2: When the functional $J(u)$ is given by (24), the structure of the neutral space is independent of the chosen $r$. Moreover, it is a $p-1$ dimensional linear subspace, coinciding with a translation of the left null space of the matrix $\Gamma w$ :

$$
\mathcal{N}_{J}(r)=r+\mathcal{N}_{J}(0)=r+\operatorname{ker}\left(w^{T} \Gamma\right) .
$$

Proof: Fix $r \in \mathbb{R}^{p}$, choose arbitrarily $\varpi \in \operatorname{ker}\left(W^{T} \Gamma\right)$ and set $\rho=r+\varpi$. Then, using the expression (25), it is straightforward to verify that

$$
\mathcal{S}_{J}(r)=\frac{1}{\phi(\theta)} \frac{w^{T} \Gamma r}{w^{T} \Gamma w}=\frac{1}{\phi(\theta)} \frac{w^{T} \Gamma(r+\varpi)}{w^{T} \Gamma w}=\mathcal{S}_{J}(\rho) .
$$

\section{DESIGN OF THE ERROR-FEEDBACK CONTROLLER}

As explained in the previous sections, given $\tilde{u} \in \tilde{U}$ that provides a well-defined value of $J(\tilde{u}, r)$, it is possible to add any element of $u^{\prime} \in \mathcal{U}_{0}$ and still obtain the same value $J(\tilde{u}+$ $\left.u^{\prime}, r\right)=J(\tilde{u}, r)$. This fact can be exploited for designing an error-feedback controller, which will allow guaranteeing some transient specifications, e.g. convergence of the state 
trajectory to the steady state with a desired decay ratio. More specifically, let us consider the input $u(t)$ as follows

$$
u=\frac{\alpha}{\phi(\theta)}+K(\theta)(x-\alpha k)
$$

Then, the dynamics of $e_{x}(t)=x(t)-\alpha k$ will obey

$$
\dot{e_{x}}(t)=A(\theta) x+b(\theta) \frac{\alpha}{\phi(\theta)}+b(\theta) K(\theta) e_{x}
$$

which, by considering the Lyapunov function (12), leads to

$$
\begin{gathered}
\dot{\Psi}(x, \theta)=e_{x}^{T}\left(A(\theta)^{T} P(x, \theta)+P(x, \theta) A(\theta)+\dot{P}(x, \theta)+\ldots\right. \\
\left.\ldots K(\theta)^{T} b(\theta)^{T} P(x, \theta)+P(x, \theta) b(\theta) K(\theta)\right) e_{x}
\end{gathered}
$$

In order to guarantee a decay ratio $\alpha$, the following must be ensured $\dot{\Psi}(x, \theta)<\alpha \Psi(x, \theta)$ which, taking into account (32), means

$$
\begin{aligned}
& A(\theta)^{T} P(x, \theta)+P(x, \theta) A(\theta)+\dot{P}(x, \theta)-\alpha P(x, \theta) \\
& +K(\theta)^{T} b(\theta)^{T} P(x, \theta)+P(x, \theta) b(\theta) K(\theta)<0
\end{aligned}
$$

If both $P(x, \theta)$ and $K(\theta)$ are unknown, the condition (33) represents an infinite number of bilinear matrix inequalities (BMIs). In order to convert (33) into a more easily tractable linear matrix inequality (LMI) form, let us consider the dual form obtained by pre- and post-multiplying it by $Q(x, \theta)=$ $P(x, \theta)^{-1}[14]$ and, for the sake of simplicity, let us consider $P(x, \theta)=P$, i.e. $Q(x, \theta)=Q$ [2], thus obtaining

$$
Q A(\theta)^{T}+A(\theta) Q-\alpha Q+Q K(\theta)^{T} b(\theta)^{T}+b(\theta) K(\theta) Q<0
$$

which, using the auxiliary variable $\Gamma(\theta)=K(\theta) Q$ converts into

$$
Q A(\theta)^{T}+A(\theta) Q-\alpha Q+\Gamma K(\theta)^{T} b(\theta)^{T}+b(\theta) \Gamma(\theta)<0
$$

Then, (35) can be brought to an equivalent finite set of LMIs either by considering a gridding approach or a polytopic representation for $A(\theta)$ [16], in which case $b(\theta)$ needs to be a constant vector. Once the solution has been found, the controller gain can be easily recovered as $K(\theta)=$ $\Gamma(\theta) Q^{-1}$.

\section{NUMERICAL Simulations}

Let us consider the plant $\Sigma(\theta)=(A(\theta), b(\theta), C)$ with

$$
\begin{array}{cc}
A(\theta)=-\left[\begin{array}{cc}
3 \theta & 2 \theta^{2} \\
\theta & \theta^{2}
\end{array}\right], & b(\theta)=\left[\begin{array}{c}
3+14 \theta \\
1+7 \theta
\end{array}\right], \\
C=I_{2 \times 2}, & \Theta=\left[\frac{1}{2}, \frac{7}{2}\right]
\end{array}
$$

It can be proved that the positive definite matrix

$$
P=\left[\begin{array}{cc}
3.750 & -6.250 \\
-6.250 & 17.500
\end{array}\right]
$$

fulfills the Lyapunov equation

$$
A(\theta)^{T} P+P A(\theta)<0 \quad \forall \theta \in \Theta,
$$

this guaranteeing quadratic stability of the system. Moreover the identity

$$
-[A(\theta)]^{-1} b(\theta)=\left[\begin{array}{l}
\frac{1}{\theta} \\
\frac{7}{\theta}
\end{array}\right]=\left[\begin{array}{l}
1 \\
7
\end{array}\right] \frac{1}{\theta}
$$

in in force, so that (10) holds with $k=\left[\begin{array}{ll}1 & 7\end{array}\right]^{T}, \phi(\theta)=\theta^{-1}$. As the system is Steady Stable, and $C(\theta)$ is a constant matrix, the optimal input for the functional (4) can be found using the results of section IV.

Consider the constant reference vector $r=\left[\begin{array}{ll}4 & 5\end{array}\right]^{T}$. According to (18), the solution for (17) is given by

$$
\begin{aligned}
u^{*}(t) & =\theta(t) \frac{1}{50}\left[\begin{array}{ll}
1 & 7
\end{array}\right]\left[\begin{array}{l}
4 \\
5
\end{array}\right]=\frac{39}{50} \theta(t)= \\
& =\frac{39}{50}\left(2+\frac{1}{2} \sin t\right)=\alpha^{*} \frac{1}{\phi(\theta)}
\end{aligned}
$$

with $\alpha^{*}=39 / 50$.

In the simulations, the scheduling parameter has been assumed to obey the evolution law $\theta(t)=2+\frac{1}{2} \sin t$. We point out that the knowledge of such law is not used in the controller design. As a comparison, the superposition $u_{f}^{*}(t)$ of the optimized feedforward input $u^{*}(t)$ with an error feedback stabilizing controller $K(\theta)\left(x-\alpha^{*} k\right)$ is also considered.

In Fig. 2 the behavior of the optimal input $u^{*}(t)$ is shown together with the one of $u_{f}^{*}(t)$ and in Fig. 3 it is depicted the output of the system driven, respectively, by $u^{*}$ and by $u_{f}^{*}$. As expected for a Steady Stable plant, one has $y(t) \rightarrow y_{\infty}$.

In Fig. 4 optimal output trajectories $y(t)$ and $y_{f}(t)$, corresponding to the inputs $u^{*}$ and $u_{f}^{*}$ respectively, are plotted in the output space $\mathbb{R}^{p}$ together with the reference vector $r$ and the line of reachable steady output $y_{\infty}=\alpha k$. It is clearly visible that the limit point of the path coincides with the projection of the reference $r$ onto the $\alpha k$ line.

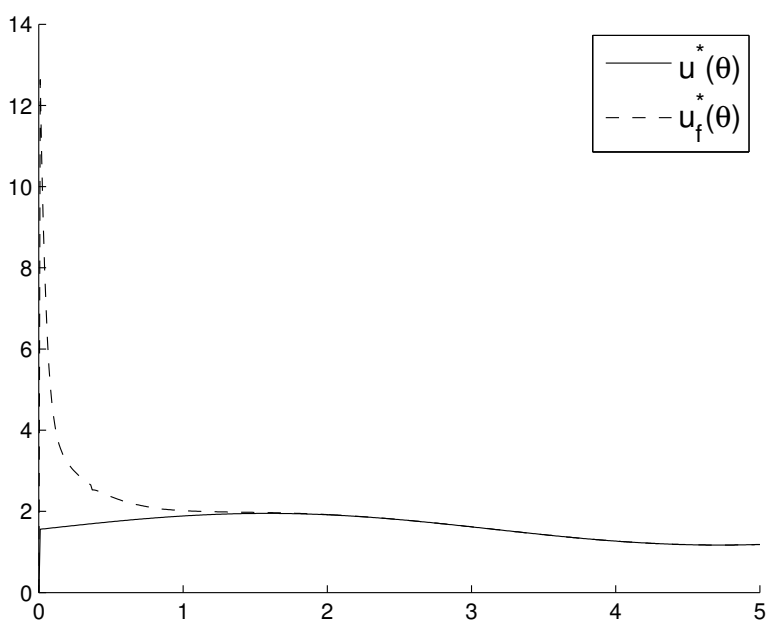

Fig. 2. Optimal inputs $u^{*}$ (pure feedforward) and $u_{f}^{*}$ (feedback and feedforward) for the numerical simulation with squared euclidean distance 

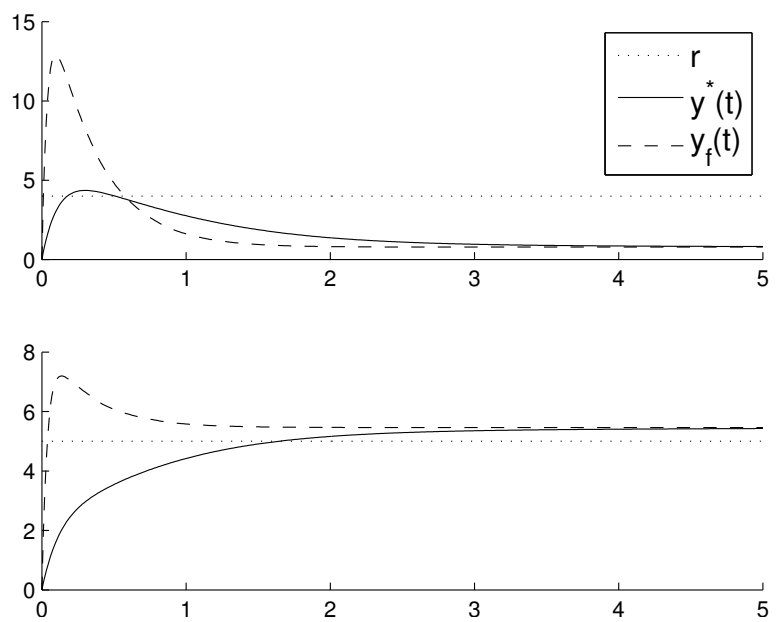

Fig. 3. Optimal output responses $y(t)$ and $y_{f}(t)$ for the numerical simulation with squared euclidean distance

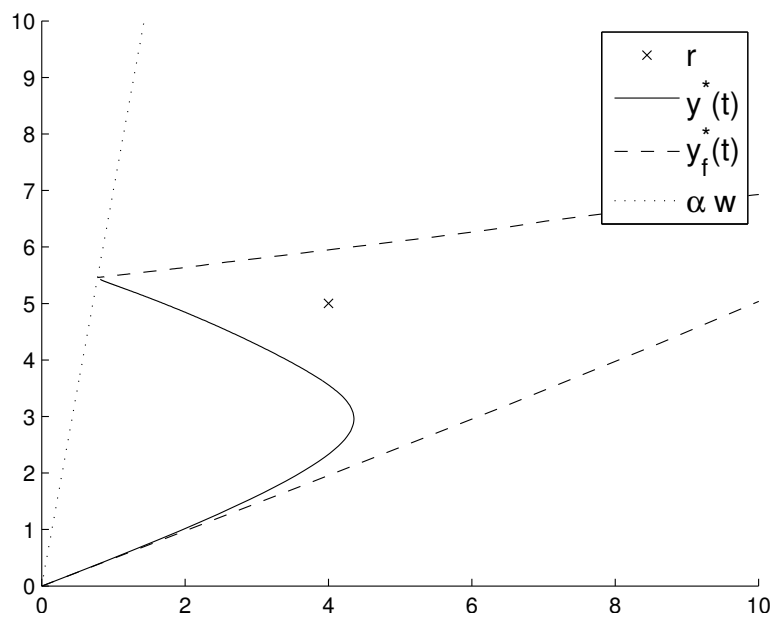

Fig. 4. Optimal output trajectories $y^{*}(t)$ and $y_{f}^{*}(t)$ in the output space, with the achievable steady output line $\alpha k$ and the reference vector $r$

\section{REFERENCES}

[1] A. Abdullah and M. Zribi. Model reference control of LPV systems. Journal of the Franklin Institute, 346:854-871, 2009.

[2] G. Becker, A. Packard, D. Phillbrick, and G. Balas. Control of parametrically-dependent linear systems: a single quadratic Lyapunov approach. In Proceedings of the American Control Conference, pages 2795-2799, 1993

[3] K. A. Bordingnon and W. C. Durham. Closed-form solutions to constrained control allocation problem. Journal of Guidance, Control, and Dynamics, 18(5):1000-1007, 1995.

[4] A. Casavola and E. Garone. Fault-tolerant adaptive control allocation schemes for overactuated systems. International Journal of Robust and Nonlinear Control, 20(17):1958-1980, 2010.

[5] D. Corona and A. Cristofaro. Some remarks on optimal output regulation for weakly dual redundant plants. In Control and Automation (MED), 2016 24th Mediterranean Conference on, pages 1205-1211, 2016

[6] D. Corona, A. Cristofaro, and M.L. Corradini. Optimal output regulation for underactuated systems with quasiperiodic references. IFAC World Congress 2017, under review, 2017.

[7] A. Cristofaro and S. Galeani. Output invisible control allocation with steady-state input optimization for weakly redundant plants. In IEEE Conf. on Decision and Control, pages 4246-4253, 2014.

[8] A. Cristofaro and T.A. Johansen. Fault tolerant control allocation using unknown input observers. Automatica, 50(7):1891-1897, 2014.
[9] A. Cristofaro and L. Zaccarian. An adaptive pseudo-inverse method for the fault-tolerant output allocation in linear observers with redundant sensors. In Decision and Control (CDC), 2016 IEEE 55th Conference on, pages 5402-5407, 2016.

[10] Thor I Fossen. Handbook of marine craft hydrodynamics and motion control. John Wiley \& Sons, 2011

[11] S.A. Frost and M. Bodson. Resource balancing control allocation. In American Control Conference (ACC), 2010, pages 1326-1331, 2010.

[12] S. Galeani and S. Pettinari On dynamic input allocation for fat plants subject to multi-sinusoidal exogenous inputs. In IEEE Conf. on Decision and Control, pages 2396-2403, 2014.

[13] S. Galeani, A. Serrani, G. Varano, and L. Zaccarian. On linear overactuated regulation using input allocation. In IEEE Conf. on Decision and Control, pages 4471-4476, Orlando (FL), USA, Dec., 12-15 2011.

[14] R. Goebel, T. Hu, and A. R. Teel. Dual matrix inequalities in stability and performance analysis of linear differential/difference inclusions. In L. Menini, L. Zaccarian, and C. T. Abdallah, editors, Current Trends in Nonlinear Systems and Control. Birkhäuser Boston, 2006.

[15] O. Härkegård. Dynamic control allocation using constrained quadratic programming. Journal of Guidance, Control, and Dynamics, 27(6):1028-1034, 2004.

[16] C. Hoffmann and H. Werner. A survey of linear parameter-varying control applications validated by experiments or high-fidelity simulations. IEEE Transactions on Control Systems Technology, 23(2):416433, 2015.

[17] T. A. Johansen and T. I. Fossen. Control allocation - a survey. Automatica, pages 1087-1103, 2013

[18] A. Kwiatkowski, M.-T. Boll, and H. Werner. Automated Generation and Assessment of Affine LPV Models. In Proceedings of the 45th IEEE Conference on Decision and Control, pages 6690-6695, 2006.

[19] S. Moe, W. Caharija, K. Y. Pettersen, and I. Schjolberg. Path following of underactuated marine surface vessels in the presence of unknown ocean currents. In American Control Conference (ACC), 2014, pages 3856-3861, 2014

[20] S. Moe, A.R. Teel, G. Antonelli, and K. Y. Pettersen. Stability analysis for set-based control within the singularity-robust multiple task-priority inverse kinematics framework. In IEEE Conf. on Decision and Control, pages 171-178, 2015.

[21] A. P. Molchanov and Y. S. Pyatnitskiy. Criteria of asymptotic stability of differential and difference inclusions encountered in control theory. Systems and Control Letters, 13:59-64, 1989.

[22] M. Q. Nguyen, J. M. Gomes da Silva Jr., O. Sename, and L. Dugard. A state feedback input constrained control design for a 4-semi-active damper suspension system: a quasi-LPV approach. In Proceedings of the 8th IFAC Symposium on Robust Control Design (ROCOND), pages 259-264, 2015 .

[23] A. Pavlov, N. van de Wouw, and A. Nijmeijer. Convergent systems: analysis and synthesis. In T. Meurer, K. Graichen, and E. D. Gilles, editors, Control and observer design for nonlinear finite and infinite dimensional systems. Springer, 2005.

[24] R. H. Rogne, T. A. Johansen, and T. I. Fossen. Observer and imubased detection and isolation of faults in position reference systems and gyrocompasses with dual redundancy in dynamic positioning. In Control Applications (CCA), 2014 IEEE Conference on, pages 83-88, 2014.

[25] D. Rotondo, F. Nejjari, and V. Puig. Quasi-LPV modeling, identification and control of a twin rotor MIMO system. Control Engineering Practice, 21(6):829-846, 2013.

[26] D. Rotondo, V. Puig, F. Nejjari, and J. Romera. A fault-hiding approach for the switching quasi-LPV fault-tolerant control of a four-wheeled omnidirectional mobile robot. IEEE Transactions on Industrial Electronics, 62(6):3932-3944, 2015.

[27] M. Sassano and L. Zaccarian. A hybrid anti-windup scheme for output saturated SISO linear closed loops. In Decision and Control (CDC), 2013 IEEE 52nd Annual Conference on, pages 2647-2652, 2013.

[28] A Serrani. Output regulation for over-actuated linear systems via inverse model allocation. In IEEE Conf. on Decision and Control, pages 4871-4876, 2012.

[29] J. S. Shamma. An overview of LPV systems. In J. Mohammadpour and C. Scherer, editors, Control of Linear Parameter Varying Systems with Applications. Springer, 2012.

[30] J. Tjønnås and T. A. Johansen. Optimizing adaptive control allocation with actuator dynamics. In IEEE Conf. on Decision and Control, pages 3780-3785, 2007.

[31] L. Zaccarian. Dynamic allocation for input-redundant control systems. Automatica, 45:1431-1438, 2009.

[32] J. Zhou, M. Canova, and A. Serrani. Predictive inverse model allocation for constrained over-actuated linear systems. Automatica, 67:267-276, 2016. 